Technical Note

\title{
Effect of Changing Phantom Thickness on Helical Radiotherapy Plan: Dosimetric Analysis
}

\author{
Sibel KARACA ${ }^{1, a}$ \\ ${ }^{\text {I}}$ Erzurum Regional Training and Research Hospital, Erzurum, Turkey \\ ${ }^{a}$ E-mail address: sibeltuzlaci@gmail.com
}

(received 1 November 2018; revised 27 December and 7 February 2019; accepted 3 April 2019)

\begin{abstract}
Purpose: The aim of this study is to investigate the effect of changing phantom thickness on high dose region of interest (HD_ROI) and low dose ROI's (LW_ROI's) doses during helical radiotherapy (RT) by utilizing Adaptive RT (ART) technique.

Materials and Methods: The cylindrical phantom (CP) is wrapped with different thickness boluses and scanned in the kilovoltage computed tomography (KVCT). HD_ROI and LW_ROI's were created in contouring system and nine same plans $(1.8 \mathrm{~Gy} / \mathrm{Fr})$ were made with images of different thicknesses $\mathrm{CP}$. The point dose measurements were performed using ionization chamber in Helical Tomotherapy (HT) treatment machine. For detecting thickness reduction effect, CP was irradiated using bolus-designed plans and it was irradiated using without bolus plan. The opposite of this scenario was applied to determine the thickness increase. KVCT and megavoltage CT (MVCT) images were used for dose comparison. The HT Planned Adaptive Software was used to see the differences in the planning and verification doses at dose volume histograms (DVH).

Results: Point dose measurements showed a $4.480 \%$ dose increase in $0.5 \mathrm{~cm}$ depth reduction for HD_ROI. These differences reached $8.508 \%$ in $2 \mathrm{~cm}$ depth and $15,279 \%$ in $5 \mathrm{~cm}$ depth. At the same time, a dose reduction of $0.665 \%$ was determined for a $0.5 \mathrm{~cm}$ depth increase, a dose reduction of $1.771 \%$ was determined for a $2 \mathrm{~cm}$ depth increase, a dose reduction of $5.202 \%$ was determined for a $5 \mathrm{~cm}$ depth increase for the HD_ROI. The ART plan results show that the dose changes in the HD_ROI was greater than the LW_ROI's.

Conclusion: Phantom thicknesses change can lead to a serious dose increase or decrease in the HD_ROI and LW_ROI's.
\end{abstract}

Key words: phantom thickness; adaptive radiotherapy; dissymmetric analysis; helical tomotherapy.

\section{Introduction}

Anatomical changes during radiotherapy (RT) are very common [1]. Dosimetric differences may occur between the planned site and the irradiated site, especially for prolonged treatment [2]. Weight loss or gain, swelling due to biliary movements, possible postoperative edema, changes in respiration-induced motion and positional changes can lead to contour changes. This change in distance between the skin and the treatment area also leads to a changing in dose homogeneity $[3,4]$. The changes in the anatomy affect on the shape of the distribution of the delivered dose and result in potential differences between treatment doses and planned doses $[5,6]$.

Changes in body contour during RT can affect the dose distribution in the tumor and critical organs, which can be a cause of recurrence or late toxicities [7,8]. All these changes during treatment may be clinically relevant [9] and these can result in the need to recalculate treatment plan to obtain actual dose distribution [10-12]. Such dosimetric problems can be easily observed with the developing technology [13].

Helical tomotherapy (HT) (TomoTherapy Inc, Madison, WI) is an RT device that features image-guided RT (IGRT) through a linear accelerator and helical megavoltage computer tomography (MVCT) scanner $[14,15]$. HT machine combines a straight $6 \mathrm{MV}$ linear accelerator mounted on a ring gantry with CT technology for image-guided intensity-modulated radiation therapy (IG-IMRT) treatment [16-18]. IG-IMRT allows verification of patient positioning, target, tumor/organ registration to assess internal motion (geometric shift, and shape/volume changes), and reconstruction of delivered dose [19]. The IG process is performed in two steps: (i) patient positioning, MVCT scan, automatic fusion (registration) of the planning KVCT (ii) an inspection by the radiation therapists of the resulting match as per instructions of the treating physician and manual correction shifts [20]. 
The IGRT method has no potential to correct organ movement, setup failures, correct organ movement, correct potential changes in the dose, patient contour change or dosimetric errors. So adaptive radiotherapy (ART) has developed to overcome similar problems. ART is required in the fractions after initiation of treatment and the patient first-day treatment plan may be replaced by ART [21-23]. Daily recorded MVCT images were the basis of ART [24,25]. The MVCT scan contrast is linear with respect to the electron density of material imaged. This significant result demonstrated that the MVCT images are suitable for RT dose calculations in addition to IG of patient position [5,25]. ART involves replanning and repeated imaging during the course of treatment [23]. ART permits verification of target and critical organs registration, patient positioning to assess internal motion volume changes, geometric shift and it could be applied to reduce dose to critical organs and eventually to improve quality of life [26-28].

In this study, dosimetric changes due to $\mathrm{CP}$ thickness variation using bolus method at mid-site high dose region of interest (HD_ROI) and low dose region of interests (LW_ROI1, LW_ROI2, and LW_ROI3) were analyzed using point dose measurements and ART software planning. In fact, volumetric changes in patients under normal conditions are typically asymmetric, not symmetric. This situation was ignored in the study. A visual simulation experiment was tried to be made and it was tried to show the importance of the potential volumetric change effect of the dose.

\section{Materials and Methods}

\section{Phantom preparation and volume definition}

The water equivalent cylindrical (cheese) phantom is (CP) used for the delivery quality assurance (DQA) in HT device. HT (Accuray, Madison, WI) cheese phantom is a cylinder of $15 \mathrm{~cm}$ in radius and $15 \mathrm{~cm}$ in length. (Figure 1). The $\mathrm{CP}$ is made up of two equal parts. It has along the other direction a series of holes for placing ion chambers. The distance between the holes is $1 \mathrm{~cm}$ (one hole is set $0.5 \mathrm{~cm}$ from the central) and allows ionization chambers for point measurements. In this study, point dose measurements were made using an ion chamber. Ion-chamber measurements can be performed at the same time by considering both the coronal and sagittal plane.

The bolus is a soft, resilient and approximating tissueequivalence material which is used for increasing a $\mathrm{CP}$ thickness. In this study, Super flap Bolus Material (Density: $\left.1.02 \mathrm{~g} / \mathrm{cm}^{3}\right)$ is used. The CP wrapped with different thickness boluses $(0,0.5,1,1.5,2,2.5,3,4$ and $5 \mathrm{~cm})$, the ion chamber was placed in a hole $0.5 \mathrm{~cm}$ below the center of $\mathrm{CP}$ and it was scanned with a $3 \mathrm{~mm}$ slice width in KVCT. All images obtained were transferred via network to a workstation for contouring. Four circular volumes were drawn on all KVCT images of CP. The HD_ROI and LW_ROI1, LW_ROI2 and LW_ROI3 were created in Focal contouring system (v. 4.62). The HD_ROI was a circle of $3 \mathrm{~cm}$ in radius and $474.18 \mathrm{~cm}^{3}$,
LW_ROI1 was a circle of $1.5 \mathrm{~cm}$ and $117.03 \mathrm{~cm}^{3}, \mathrm{LW}$ _ROI2 was a circle of $42 \mathrm{~cm}$ and $116.98 \mathrm{~cm}^{3}$ and LW_ROI3 was a circle of $1.5 \mathrm{~cm}$ and $208.98 \mathrm{~cm}^{3}$. The center of HD_ROI was placed $15.5 \mathrm{~cm}$ deep in the center of the CP. LW_ROI1 was placed under the target, LW_ROI2 was placed to the left of the HD_ROI, and LW_ROI3 was placed to the top of the HD_ROI (Figure 1).

\section{TomoTherapy Treatment Planning}

Dose calculations were made with HT (Accuray Inc., Madison, USA) planning system using a field width of $2.5 \mathrm{~cm}$, pitch of 0.3 on a fine calculation grid, and a modulation factor of 2 ; final dose calculations were set to be completed after 30 iterations for each different thickness CP images. The HD_ROI was planned to receive $45 \mathrm{~Gy}$ in 25 fractions $(1.8 \mathrm{~Gy} / \mathrm{Fr})$. Nine same plans were created of different thicknesses CP images. The delivery quality assurance (DQA) plans were made in the DQA planning station of HT and the point doses of the hole set $0.5 \mathrm{~cm}$ below from the central of $\mathrm{CP}$ were recorded.

\section{Dosimetric Measurement}

The CP was positioned into the HT treatment couch. The point dose measurements were performed using $0.057 \mathrm{~cm}^{3}$ Exradin A1SL (Standard Imaging, Inc., Middleton, WI, USA) ionization chamber. The ionization chamber inserted on $0.5 \mathrm{~cm}$ below the center of CT and positioned in the sagittal direction. It was connected to an eight channel electrometer (TomoElectrometer, Standard Imaging Inc., Madison, WI, USA). TomoElectrometer was used to supply ionization chamber voltages and provide readings. MVCT images were taken before treatment to determine the position of the CP. MVCT images were matched against the planning KVCT images. This method provided to minimize the dosimetric uncertainties.

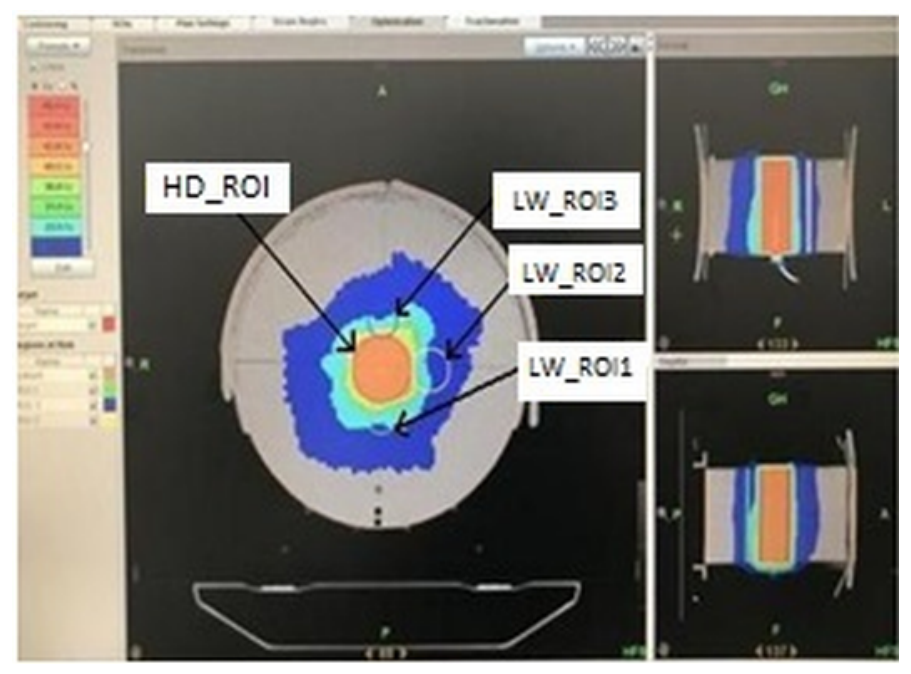

Figure 1. The cheese phantom wrapped with $1.5 \mathrm{~cm}$ bolus. Axial and saggital view of HD_ROI and LW_ ROI's and planning lines. 
The CP was irradiated with no-bolus to determine thickness reduction differences using bolus-designed plans. In addition, the $\mathrm{CP}$ was irradiated with different thick boluses to determine thickness increase differences using no-bolus planning. All measurement data were recorded and measurements were corrected by daily pressure and temperature. Calculated data was obtained as Gray (Gy) (Table 1).

\section{Planned Adaptive Radiotherapy}

ART Software (TomoTherapy Inc, Madison, WI) was used to calculate HD_ROI and LW_ROI's doses for each different thickness KVCT and MVCT images of CP. The initial plan was compared with the irradiation plans obtained at different thicknesses. Eight planning scenarios were compared:

(1) $0.5 \mathrm{~cm}$ bolus thickness irradiation plan, evaluated with the no-bolus initial plan of dose volume histograms (DVH);

(2) $1 \mathrm{~cm}$ bolus thickness irradiation plan, evaluated with the no-bolus initial plan of DVH;

(3) $1.5 \mathrm{~cm}$ bolus thickness irradiation plan, evaluated with the no-bolus initial plan of DVH;

(4) $2 \mathrm{~cm}$ bolus thickness irradiation plan, evaluated with the no-bolus initial plan of DVH;

(5) $2.5 \mathrm{~cm}$ bolus thickness irradiation plan, evaluated with the no-bolus initial plan of DVH;

(6) $3 \mathrm{~cm}$ bolus thickness irradiation plan, evaluated with the no-bolus initial plan of DVH;

(7) $4 \mathrm{~cm}$ bolus thickness irradiation plan, evaluated with the no-bolus initial plan of DVH;

(8) $5 \mathrm{~cm}$ bolus thickness irradiation plan, evaluated with the no-bolus initial plan of DVH.

The comparison of DVH with the initial planning doses (using the KVCT image of CP with no bolus) and after the irradiation (using the MVCT images of CP with $0.5,1.5$ and $5 \mathrm{~cm}$ bolus) verification doses were shown in Figure 2.

\section{Results}

In this study, point dose measurements provided a quantitative comparison. Table 1 summarizes ion chamber measurements results for HD_ROI. Point dose measurements showed a $4.480 \%$ dose increase in $0.5 \mathrm{~cm}$ depth reduction, this difference reached $8.508 \%$ in $2 \mathrm{~cm}$ depth and $15.279 \%$ in $5 \mathrm{~cm}$ depth. At the same time, a dose reduction of $0.665 \%$ was determined for a $0.5 \mathrm{~cm}$ depth increase for the target, which is $1.771 \%$ for $2 \mathrm{~cm}$ depth difference and $5.202 \%$ for $5 \mathrm{~cm}$ depth. In the DVH analysis in the ART software, Dmax difference was $0.540 \%$ in the $0.5 \mathrm{~cm}$ depth reduction of the target while this difference was $14.39 \%$ in $5 \mathrm{~cm}$ were found (Table 2). These values are between $0.441 \%$ and $7.323 \%$ for Dmax in LW_ROI (Table 3), between $0.00 \%$ and $11.492 \%$ for Dmax in LW_ROI2 (Table 4) and between $0.981 \%$ and $6.798 \%$ for Dmax in LW_ROi3 (Table 5).

\section{Discussion}

Weight changes during RT are often seen and also cause the body contour to change. There are very few studies on the dosimetric effect that may occur due to the change of the body contour [29,30]. In this study, it is observed that in the case of a decrease in bolus thickness, HD_ROI and LW_ROI's of the phantom was receiving a higher dose. Reductions in body thickness can cause an overdosage in normal tissue around the target [23,31]. Similar studies have shown that reducing the amount of bolus used leads to an increased maximum dose $[32,33]$.
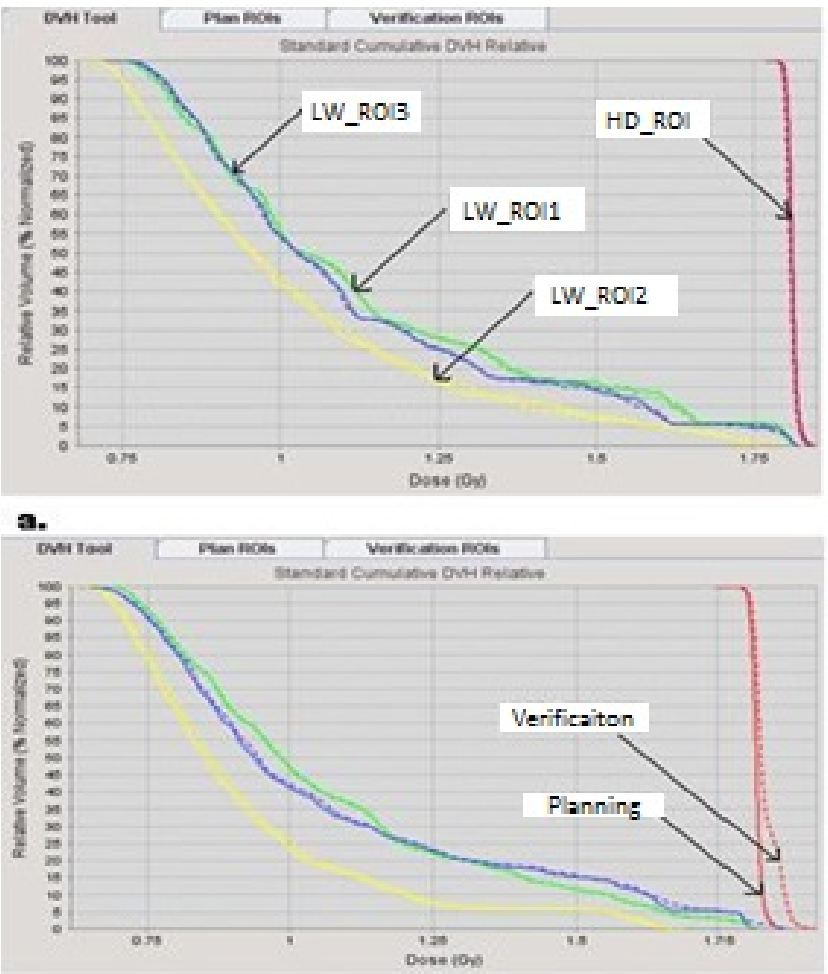

b.

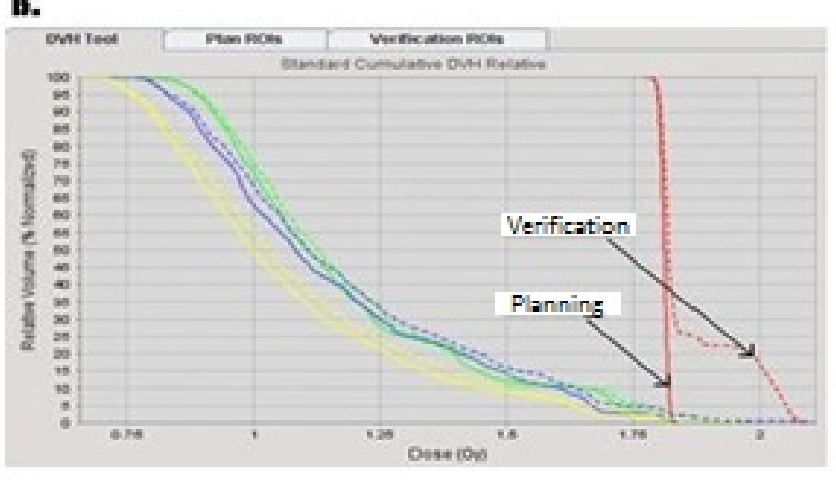

c.

Figure 2. DVH comparison of KVCT and MVCT images in ART software (MVCT (dash line) and KVCT (solid line)). (a) Verification planning with $0.5 \mathrm{~cm}$ bolus and initial planning with no bolus. (b) Verification planning with $1.5 \mathrm{~cm}$ bolus and initial planning with no bolus. (c) Verification planning with $5 \mathrm{~cm}$ bolus and initial planning with no bolus. 
Table 1. Results of ion chamber measurements for HD ROI and calculations values for different thicknesses boluses.

\begin{tabular}{|c|c|c|c|c|c|c|}
\hline $\begin{array}{l}\text { Bolus Thickness } \\
\text { (cm) }\end{array}$ & $\begin{array}{c}\text { Planning System (DQA) } \\
\text { Point Dose Values } \\
\text { (Gy)(With-Bolus) }\end{array}$ & $\begin{array}{c}\text { Ion Chamber Point } \\
\text { Dose Measuring } \\
\text { Values (Gy) } \\
\text { (No-Bolus) } \\
\end{array}$ & $\begin{array}{c}\text { Per. Dif. } \\
\%\end{array}$ & $\begin{array}{c}\text { Planning System (DQA) } \\
\text { Point Dose Values(Gy) } \\
\text { (No-Bolus) }\end{array}$ & $\begin{array}{c}\text { Ion Chamber Point } \\
\text { Dose Measuring } \\
\text { Values(Gy) } \\
\text { (With -Bolus) } \\
\end{array}$ & $\begin{array}{c}\text { Per. Dif. } \\
\%\end{array}$ \\
\hline 0.5 & 1.808 & 1.889 & 4.480 & 1.807 & 1.795 & 0.665 \\
\hline 1 & 1.817 & 1.926 & 5.998 & 1.807 & 1.788 & 1.051 \\
\hline 1.5 & 1.815 & 1.930 & 6.336 & 1.807 & 1.782 & 1.384 \\
\hline 2 & 1.810 & 1.964 & 8,508 & 1.807 & 1.775 & 1.771 \\
\hline 2.5 & 1.812 & 1.972 & 8.830 & 1.807 & 1.768 & 2.158 \\
\hline 3 & 1.813 & 1.990 & 9.763 & 1.807 & 1.747 & 3.320 \\
\hline 4 & 1.816 & 2.056 & 13.216 & 1.807 & 1.733 & 4.095 \\
\hline 5 & 1.813 & 2.090 & 15.279 & 1.807 & 1.713 & 5.202 \\
\hline
\end{tabular}

Per. Dif.: Percent Different, percentage difference $=100 *|\mathrm{a}-\mathrm{b}| / \mathrm{b}$, Gy: Gray

Table 2. Dosimetric changes on HD_ROI in ART planning.

\begin{tabular}{|c|c|c|c|c|c|c|c|c|c|}
\hline \multirow{2}{*}{$\begin{array}{c}\text { Bolus Thickness } \\
\text { (cm) }\end{array}$} & \multicolumn{3}{|c|}{ Planning } & \multicolumn{3}{|c|}{ Verification } & \multicolumn{3}{|c|}{ Per. Dif. } \\
\hline & Dmax & D50\% & D98\% & Dmax & D50\% & D98\% & Dmax & D50\% & D98\% \\
\hline 0.5 & 1.849 & 1.81 & 1.797 & 1.839 & 1.807 & 1.794 & 0.541 & 0.166 & 0.166 \\
\hline 1 & 1.975 & 1.814 & 1.791 & 1.996 & 1.82 & 1.791 & 1.063 & 0,220 & 0,000 \\
\hline 1.5 & 1.860 & 1.813 & 1.797 & 1.919 & 1.822 & 1.798 & 3.170 & 0.496 & 0,055 \\
\hline 2 & 1.849 & 1.811 & 1.815 & 1.925 & 1.823 & 1.803 & 4.110 & 0.662 & 0.661 \\
\hline 2.5 & 1.862 & 1.811 & 1.816 & 1.940 & 1.818 & 1.834 & 4.180 & 0.386 & 0.991 \\
\hline 3 & 1.847 & 1.810 & 1.795 & 1.944 & 1.808 & 1.786 & 5.252 & 0.110 & 0.501 \\
\hline 4 & 1.836 & 1.813 & 1.797 & 2.044 & 1.820 & 1.829 & 11.32 & 0.386 & 1.780 \\
\hline 5 & 1.842 & 1.813 & 1.797 & 2.107 & 1.821 & 1.797 & 14.39 & 0.441 & 0.000 \\
\hline
\end{tabular}

Dmax: Maximum absorbed dose in target, D98\%: Minimum absorbed dose covering 98\%of the volume, D50\%: Absorbed dose received by 50\%of the volume, D30\%: Absorbed dose received by $30 \%$ of the volume

Table 3. Dosimetric changes on LW_ROI1 in ART planning.

\begin{tabular}{|c|c|c|c|c|c|c|c|c|c|}
\hline \multirow{2}{*}{$\begin{array}{l}\text { Bolus Thickness } \\
(\mathrm{cm})\end{array}$} & \multicolumn{3}{|c|}{ Planning } & \multicolumn{3}{|c|}{ Verification } & \multicolumn{3}{|c|}{ Per. Dif. } \\
\hline & Dmax & D30\% & D50\% & Dmax & D30\% & D50\% & Dmax & D30\% & D50\% \\
\hline 0.5 & 1.812 & 1.212 & 1.032 & 1.804 & 1.208 & 1.037 & 0.441 & 0.330 & 0.484 \\
\hline 1 & 1.853 & 1.159 & 1.042 & 1.855 & 1.154 & 1.041 & 0.108 & 0.431 & 0.096 \\
\hline 1.5 & 1.808 & 1.156 & 0.981 & 1.817 & 1.156 & 0.984 & 0.498 & 0.000 & 0.306 \\
\hline 2 & 1.828 & 1.126 & 0.983 & 1.888 & 1.130 & 0.988 & 3.282 & 0.355 & 0.252 \\
\hline 2.5 & 1.797 & 0.851 & 0.647 & 1.862 & 0.863 & 0.656 & 3.673 & 1.410 & 1.391 \\
\hline 3 & 1.81 & 1.197 & 1.049 & 1.866 & 1.189 & 1.054 & 3.094 & 0.668 & 0.477 \\
\hline 4 & 1.825 & 1.292 & 1.152 & 1.949 & 1.325 & 1.165 & 6.795 & 2.554 & 1.128 \\
\hline 5 & 1.816 & 1.238 & 1.104 & 1.988 & 1.263 & 1.130 & 7.323 & 2.019 & 2.355 \\
\hline
\end{tabular}

Table 4. Dosimetric changes on LW_ROI2 in ART planning.

\begin{tabular}{|c|c|c|c|c|c|c|c|c|c|}
\hline \multirow{2}{*}{$\begin{array}{l}\text { Bolus Thickness } \\
\text { (cm) }\end{array}$} & \multicolumn{3}{|c|}{ Planning } & \multicolumn{3}{|c|}{ Verification } & \multicolumn{3}{|c|}{ Per. Dif. } \\
\hline & Dmax & D30\% & D50\% & Dmax & D30\% & D50\% & Dmax & D30\% & D50\% \\
\hline 0.5 & 1.816 & 1.189 & 1.025 & 1.816 & 1.185 & 1.024 & 0.000 & 0.336 & 0.098 \\
\hline 1 & 1.833 & 1.318 & 1.102 & 1.835 & 1.305 & 1.089 & 0.109 & 0.986 & 1.179 \\
\hline 1.5 & 1.811 & 1.128 & 0.934 & 1.867 & 1.137 & 0.943 & 3.092 & 0.798 & 0.964 \\
\hline 2 & 1.821 & 1.273 & 1.065 & 1.899 & 1.287 & 1.074 & 5.875 & 1.099 & 0.845 \\
\hline 2.5 & 1.825 & 0.786 & 0.595 & 1.928 & 0.808 & 0.609 & 5.643 & 2.798 & 2.352 \\
\hline 3 & 1.816 & 1.267 & 1.111 & 1.929 & 1.277 & 1.115 & 6.222 & 0.789 & 0.360 \\
\hline 4 & 1.818 & 1.241 & 1.153 & 2.020 & 1.276 & 1.165 & 11.111 & 2.820 & 1.040 \\
\hline 5 & 1.829 & 1.246 & 1.078 & 2.103 & 1.284 & 1.105 & 11.492 & 3.049 & 2.505 \\
\hline
\end{tabular}

Table 5. Dosimetric changes on LW_ROI3in ART planning.

\begin{tabular}{|c|c|c|c|c|c|c|c|c|c|}
\hline \multirow{2}{*}{$\begin{array}{l}\text { Bolus Thickness } \\
\text { (cm) }\end{array}$} & \multicolumn{3}{|c|}{ Planning } & \multicolumn{3}{|c|}{ Verification } & \multicolumn{3}{|c|}{ Per. Dif. } \\
\hline & Dmax & D30\% & D50\% & Dmax & D30\% & D50\% & Dmax & D30\% & D50\% \\
\hline 0.5 & 1.783 & 1.088 & 0.958 & 1.807 & 1.101 & 0.952 & 1.346 & 1.194 & 0.626 \\
\hline 1 & 1.834 & 1.118 & 0.960 & 1.816 & 1.118 & 0.964 & 0.981 & 0.00 & 0.417 \\
\hline 1.5 & 1,838 & 0.952 & 0.853 & 1.868 & 0.961 & 0.860 & 1.632 & 0.945 & 0.820 \\
\hline 2 & 1.831 & 1.121 & 0.960 & 1.901 & 1.136 & 0.978 & 3.823 & 1.338 & 1.875 \\
\hline 2.5 & 1.827 & 0.930 & 0.799 & 1.879 & 0.946 & 0.810 & 2.846 & 1.720 & 1.377 \\
\hline 3 & 1.816 & 1.097 & 0.965 & 1.902 & 1.104 & 0.974 & 4.735 & 0.638 & 0.932 \\
\hline 4 & 1.822 & 1.221 & 1.020 & 1.932 & 1.240 & 1.046 & 6.037 & 1.556 & 2.549 \\
\hline 5 & 1.824 & 1.134 & 0.994 & 1.948 & 1.174 & 1.026 & 6.798 & 3.527 & 3.219 \\
\hline
\end{tabular}


The HT treatment machine hasn't got any auto-segmentation tools that routinely help in finding the shapes of ROI's. Before the comparison of the planned and verification doses, the shape of the irradiated structures must be manually described on the MVCT scans. In this case, an error in the statistical parameters leads to a little increase in the calculated doses of MVCT compared to KVCT [34].

In this study, found that a reduction of $1 \mathrm{~cm}$ bolus showed a $5.998 \%$ dose increase, $1,5 \mathrm{~cm}$ bolus showed a $6.336 \%$ dose increase and $2 \mathrm{~cm}$ bolus showed an $8.508 \%$ dose increase for the HD_ROI. S Jang and C Watchman study showed that a reduction of $1 \mathrm{~cm}$ bolus showed a $9 \% \pm 2 \%$ dose reduction for the target. The ratio of planned dose to delivered dose to organs at risk (OAR's) was increased $7 \%$ by decreasing phantom Radius by 1.5 , however, lower than target regions [35]. Chow and Jiang reported that a $2 \mathrm{~cm}$ decrease in contour depth, caused by the patient body weight loss, could increase the dose to the target and normal organs by more than 5\% [33]. Pair ML et al. showed that for differences due to surface distance (SSD) change, a dose difference was found at $2.9 \%$ and $3.6 \%$ in $1 \mathrm{~cm}$ SSD change and the radiation oncology team decides whether replanning should be done if the change is $1 \mathrm{~cm}$ or more [36]. Hoon Sik Choi and his colleagues claimed that ART replanning will be needed if there is a $1.5 \mathrm{~cm}$ decrease in body contour or a $2 \mathrm{~cm}$ increase in their work [37].
Body thickness increasing can be seen in the RT process as well as reducing thickness. There is no study on the dosimetric effect of increasing body thickness when a literature review is performed. In this study, a reduction of $0.5 \mathrm{~cm}$ bolus showed a $5.480 \%$ dose increase. At the same time, an increase of $0.5 \mathrm{~cm}$ bolus showed a $0,665 \%$ dose reduction was observed. This rate of changes was similar in the other depths (Table 1). So, it was determined that the dosimetric dose difference with phantom thickness reduction was much higher than thickness increasing. This result can be considered interesting.

Finally, it has been determined that there may be significant differences in the measurement of changing depth in the HD_ROI and LW_ROI's doses. In addition, the ART plan results show that the dose increasing in the HD_ROI was greater than the LW_ROI's due to the depth reduction.

\section{Conclusion}

This study is presented as a simulation experiment to visualize of differences between doses against phantom thickness changes. Excessive changes in body thickness may result in significant dosimetric impact. Regularization of the dosimetric follow-up reveals the importance of recalculating treatment plan to obtain actual dose distribution.

\section{References}

[1] Dubben HH, Thames HD, Beck-Bornholdt HP. Tumor volume: a basic and specific response predictor in radiotherapy. Radiother Oncol. 1998;47(2):167-74.

[2] Zhao L, Wan Q, Zhou Y, et al. The role of replanning in fractionated intensity modulated radiotherapy for nasopharyngeal carcinoma. Radiother Oncol. 2011;98(1):23-7.

[3] Joon-Young J, Dae Hyun K, Cheon Woong C, et al. Analysis of Changes in Skin Dose During Weight Loss when Tomotherapy of Nasopharynx Cancer. Journal of the Korean Magnetics Society. 2016;26(3):99-104.

[4] Yan D, Lockman D, Martinez A, et al. Computed tomography guided management of interfractional patient variation. Semin Radiat Oncol. 2005;15(3):168-79.

[5] Piotrowski T, Kazmierska J, Sokołowski A, et al. Impact of the spinal cord position uncertainty on the dose received during head and neck helical tomotherapy. J Med Imaging Radiat Oncol. 2013;57(4):503-511.

[6] Piotrowski T, Ryczkowski A, Adamczyk M, Jodda A. Estimation of the planning organ at risk volume for the lenses during radiation therapy for nasal cavity and paranasal sinus cancer. J Med Imaging Radiat Oncol. 2015;59(6):743-750.

[7] Han C, Chen YJ, Liu A, et al.. Actual dose variation of parotid glands and spinal cord for nasopharyngeal cancer patients during radiotherapy. Int J Radiat Oncol Biol Phys. 2008;70(4):1256-1262.

[8] Nishi T, Nishimura Y, Shibata T, et al. Volume and dosimetric changes and initial clinical experience of a two-step adaptive intensity modulated radiation therapy (IMRT) scheme for head and neck cancer. Radiother Oncol. 2013;106(1):85-89.

[9] Woodford C, Yartsev S, Dar AR, et al. Adaptive radiotherapy planning on decreasing gross tumor volumes as seen on megavoltage computed tomography images. Int J Radiat Oncol Biol Phys. 2007;69(4):1316-22.

[10] Chen C, Fei Z, Chen L, et al. Will weight loss cause significant dosimetric changes of target volumes and organs at risk in nasopharyngeal carcinoma treated with Intensity-Modulated Radiation Therapy? Med Dosim. 2014;39(1):34-37.

[11] Beltran M, Ramos M, Rovira JJ, et al. Dose variations in tumor volumes and organs at risk during IMRT for head and neck cancer. J Appl Clin Med Phys. 2012;13(6):3723.

[12] Bhide SA, Davies M, Burke K, et al. Weekly volume and dosimetric changes during chemoradiotherapy with intensity-modulated radiation therapy for head and neck cancer: A prospective observational study. Int J Radiat Oncol Biol Phys. 2010;76(5):1360-8. 
[13] Tariq I, Chen T, Kirkby NF, Jena R. Modelling and Bayesian adaptive prediction of individual patients' tumour volume change during radiotherapy. Phys Med Biol. 2016;61(5):2145-61.

[14] Fenwick JD, Tomé WA, Kissick MW, Mackie TR. Modelling simple helically delivered dose distributions. Phys Med Biol. 2005; 50(7):1505-17.

[15] Schirm M, Yartsev S, Bauman G, et al. Consistency check of planned adaptive option on helical tomotherapy. Technol Cancer Res Treat. 2008;7(6):425-32.

[16] Sen A, West MK. Commissioning experience and quality assurance of helical tomotherapy machines. J Med Phys. 2009;34(4):194-9.

[17] Olivera GH, Shepard DM, Ruchala K et al.Tomotherapy. Van Dyk J, ed. The Modern Technology of Radiation Oncology. Madison, WI: Medical Physics Publishing, 1999:521-87.

[18] Mackie TR. History of tomotherapy. Phys Med Biol. 2006;51(13):R427-53.

[19] Welsh JS, Lock M, Harari PM, et al. Clinical implementation of adaptive helical tomotherapy: a unique approach to image-guided intensity modulated radiotherapy. Tech Cancer Res Treat. 2006;5:465-479.

[20] Piotrowski T, Kaczmarek K, Bajon T, et al. Evaluation of Image-Guidance Strategies for Prostate Cancer. Technol Cancer Res Treat. 2014;13(6):583-591.

[21] Yan D. Adaptive radiotherapy: merging principle into clinical practice. Semin Radiat Oncol. 2010;20(2):79-83.

[22] Thörnqvist S, Hysing LB, Tuomikoski L, et al. Adaptive radiotherapy strategies for pelvic tumors a systematic review ofclinical implementations. Acta Oncol.2016 Aug;55(8):943-58.

[23] Surucu M, Shah KK, Roeske JC, et al. Adaptive radiotherapy for head and neck cancer implications for clinical and dosimetry outcomes. Technol Cancer Res Treat. 2017;16(2):218-223.

[24] Yadav P, Tolakanahalli R, Rong Y, Paliwal BR. The effect and stability of MVCT images on adaptive TomoTherapy. J Appl Clin Med Phys. 2010;11(4):3229.

[25] Meeks SL, Harmon JF Jr, Langen KM, et al. Performance characterization of megavoltage computed tomography imaging on a helical tomotherapy unit. Med Phys. 2005;32(8):2673-81.

[26] Welsh JS, Lock M, Harari PM, et al. Clinical Implementation of Adaptive Helical Tomotherapy: A Unique Approach to ImageGuided Intensity Modulated Radiotherapy Technology in Cancer Research and Treatment. Technol Cancer ResTreat. 2006;5(5):46579.

[27] Yan D, Liang J. Expected treatment dose construction and adaptive inverseplanning optimization: implementation for offline head and neck cancer adaptive radiotherapy. Med Phys. 2013;40(2):021719.

[28] Schwartz DL. Current progress in adaptive radiation therapy for head and neckcancer. Curr Oncol Rep. 2012;14(2):139-47.

[29] van der Horst A, Houweling AC, van Tienhoven G, et al. Dosimetric effects of anatomical changes during fractionated photon radiation therapy in pancreatic cancer patients. J Appl Clin Med Phys. 2017;18(6):142-151.

[30] Ren G, Xu S-P, Du L, et al. Actual Anatomical and Dosimetric Changes of Parotid Glands in Nasopharyngeal Carcinoma Patients during Intensity Modulated Radiation Therapy. BioMed Res Int. 2015;2015:670327.

[31] Duma MN, Kampfer S, Schuster T, et al. Adaptive radiotherapy for soft tissue changes during helical tomotherapy for head and neck cancer. Strahlenther Onkol. 2012;188(3):243-7.

[32] Ashburner MJ, Tudor S. The optimization of superficial planning target volumes (PTVs) with helical tomotherapy. J Appl Clin Med Phys. 2014;15(6):4560.

[33] Chow JCL, Jiang R. Comparison of dosimetric variation between prostate IMRT and VMAT due to patient's weight loss: Patient and phantom study. Rep Pract Oncol Radiother. 2013;18(5):272-278.

[34] Piotrowski T, Gintowt K, Jodda A, et al. Impact of the intra- and inter-observer variability in the delineation of parotid glands on the dose calculation during head and neck helical tomotherapy. Technol Cancer Res Treat. 2015;14(4):467-474.

[35] Jang S, Watchman C. SU-FF-T-596: Dosimetric Impact of Anatomic Changes Due to Patient Weight Loss On TomoTherapy Plan. Med Phys. 2009;36:2661-2662.

[36] Pair ML, Du W, Rojas HD, et al.. Dosimetric effects of weight loss or gain during volumetric modulated arc therapy and intensitymodulated radiation therapy for prostate cancer. Med Dosim. 2013;38(3):251-4.

[37] Choi HS, Jo GS, Chae JP, et al. Defining the Optimal Time of Adaptive Replanning in Prostate Cancer Patients with Weight Change during Volumetric Arc Radiotherapy: A Dosimetric and Mathematical Analysis Using the Gamma Index. Computational and Mathematical Methods in Medicine. 2017;2017. 